\title{
ESTIMATES FOR SOME KAKEYA-TYPE MAXIMAL OPERATORS
}

\author{
JOSE BARRIONUEVO
}

\begin{abstract}
We use an abstract version of a theorem of Kolmogorov-SeliverstovPaley to obtain sharp $L^{2}$ estimates for maximal operators of the form:

$$
\mathscr{M}_{\mathscr{B}} f(x)=\sup _{x \in S \in \mathscr{B}} \frac{1}{|S|} \int_{S}|f(x-y)| d y .
$$

We consider the cases where $\mathscr{B}$ is the class of all rectangles in $\mathbf{R}^{n}$ congruent to some dilate of $[0,1]^{n-1} \times\left[0, N^{-1}\right]$; the class congruent to dilates of $\left[0, N^{-1}\right]^{n-1} \times[0,1] ;$ and, in $\mathbf{R}^{2}$, the class of all rectangles with longest side parallel to a particular countable set of directions that include the lacunary and the uniformly distributed cases.
\end{abstract}

\section{INTRODUCTION}

Let $\mathscr{B}$ be a family of bounded open sets in $\mathbf{R}^{n}$ containing the origin. For a locally integrable function $f$, we define the maximal operator associated to $\mathscr{B}$ by

$$
\mathscr{M}_{\mathscr{B}} f(x)=\sup _{S \in \mathscr{B}} \frac{1}{|S|} \int_{S}|f(x-y)| d y
$$

The cases where $\mathscr{B}$ is either the family of all rectangles in $\mathbf{R}^{n}$ with prescribed eccentricity or the family of all rectangles having longest side parallel to a given set of directions, are of particular interest and have been studied by many authors.

The techniques used to prove such theorems are divided into two categories. One is the use of very delicate geometric arguments, that is, covering lemmas, and is found in [Cor 1-4, Str 1-2, F]. The other makes use of the Fourier transform ( $g$-functions, Littlewood-Paley theory) and can be found, for example, in [NSW, CDR, and CF]. In this paper we use a variant of a theorem of Kolmogorov-Seliverstov and Paley to obtain sharp $L^{2}$ estimates for certain maximal operators that include the ones studied in the above references. Arguments of this type have previously been applied to geometric maximal operators in [S1, Ha]. In particular, we obtain simpler proofs of some of these results. We prove the following:

Theorem A. For $N \geq 2$, let $\mathscr{R}_{N}$ denote the class of all rectangles in $\mathbf{R}^{n}$ congruent to some dilate of $[0,1]^{n-1} \times\left[0, N^{-1}\right]$. For a locally integrable function

Received by the editors October 29, 1990.

1980 Mathematics Subject Classification (1985 Revision). Primary 42B25, 47A65. 
$f$, let

$$
M_{N} f(x)=\sup _{x \in R \in \mathscr{R}_{N}} \frac{1}{|R|} \int_{R}|f(y)| d y .
$$

Then $\left\|M_{N} f\right\|_{L^{2}\left(\mathbf{R}^{n}\right)} \leq C_{n}(\log N)\|f\|_{L^{2}\left(\mathbf{R}^{n}\right)}$.

When $n=2$, this is Theorem 1 in [Cor 1], which is also a consequence of a result of Strömberg in [Str 1], both obtained by covering lemma arguments.

We also consider the following more singular operator in $\mathbf{R}^{n}$.

Theorem B. If we let $\widetilde{\mathscr{R}}_{N}$ denote the class of all rectangles congruent to some dilate of $[0,1] \times\left[0, N^{-1}\right]^{n-1}$ and define $\widetilde{M}_{N}$ as in $(0.2)$ we obtain

$$
\left\|\widetilde{M}_{N} f\right\|_{L^{2}\left(\mathbf{R}^{n}\right)} \leq C_{n} N^{(n-2) / 2}(\log N)\|f\|_{L^{2}\left(\mathbf{R}^{n}\right)} .
$$

An estimate like (0.3) has also been obtained in [CDR], but our argument is simpler.

In $\mathbf{R}^{2}$ we obtain a stronger result: Let $\left\{w_{j}\right\}_{j=1}^{\infty}$ be a lacunary set of directions in the plane (see $\S 3$ for the precise definition) and for a fixed $N \gg 1$ and $k=1, \ldots, N$, let $w_{j k}$ be uniformly distributed directions between $w_{j}$ and $w_{j+1}$. We form the maximal operator

$$
\mathscr{M}_{N} f(x)=\sup _{x \in R} \frac{1}{|R|} \int_{R}|f(y)| d y
$$

where the sup is taken over all rectangles in $\mathbf{R}^{2}$ with largest sides parallel to some $w_{j k}$. We prove

Theorem C. $\left\|\mathscr{M}_{N} f\right\|_{L^{2}\left(\mathbf{R}^{2}\right)} \leq C(\log N)\|f\|_{L^{2}\left(\mathbf{R}^{2}\right)}$ with $C$ independent of $N$ and $f$.

By an argument similar to the one in [Cor 1 and Str 1], Cordoba has proved in [Cor 3] a weak-type $(2,2)$ inequality for the operator $\mathscr{M}_{N}$.

The proof of Theorem $\mathrm{C}$ contains as consequences, the results in [Str 1, Str 2] treating the lacunary and uniformly distributed case separately. The lacunary case has also been proved by Stein [S1] for $p=2$ using the same ideas and was extended to $1<p \leq \infty$ by Fourier transform methods in [NSW].

Using the trivial $L^{\infty}$ estimates, together with the M. Riesz interpolation theorem, we obtain the $L^{p}$ results for $p \geq 2$.

The advantage of the method used here is that the geometry involved in the proofs is very simple compared with the original covering lemmas arguments. Also, these covering lemmas only give weak-type $(2,2)$ estimates and the strong type inequalities are then obtained by interpolating with cruder estimates for $p<2$. This does not work for Theorem $\mathrm{C}$ since there is no $L^{p}$ result for $\mathscr{M}_{N}$ for $p<2$. On the other hand, our method is essentially $L^{2}$, which is very restrictive.

The paper is organized as follows:

In $\S 1$, using the ideas in [S1], we prove an abstract form of a theorem of Kolmogorov which will be the general set up for proving the above maximal theorems. The general idea here is to linearize the operators and then use the Hilbert space structure of $L^{2}$ to obtain some algebraic inequalities that imply the boundedness of the maximal operator. 
In $\S 2$ we prove some simple estimates for convolutions in $\mathbf{R}^{n}$ that contain all the geometry necessary in the proof of the maximal theorems.

Finally in $\S 3$ we, after making some simplifications, apply the results of the preceding chapters to prove the above theorems.

In the rest of this paper $C$ (or $C_{1}, C_{2}, \ldots$ ) will denote a constant not necessarily the same on each occurrence.

This paper is based on the author's Ph.D. thesis [B], written at the University of Rochester under the direction of Allan Greenleaf.

\section{THE ABSTRACT SETTING}

We will now describe a general method for proving $L^{2}$ estimates for maximal operators that will be used in $\S 3$ to prove Theorems A, B and C. These ideas were first used by Kolmogorov and Seliverstov (see [KS1, KS2] and also [Z, vol. II, p. 161]) and later by Paley to establish some results on pointwise convergence of Fourier series. They were later extended by E. M. Stein to different problems in harmonic analysis. The following proposition is an abstract form of these (see also [S1, Ha]). We will, for simplicity, consider only the selfadjoint case that will be sufficient for our purposes though simple modifications allow one to treat nonselfadjoint operators.

Proposition 1.1. Let $A$ be a countable set and let $\left\{T_{\mu}\right\}_{\mu \in A}$ be a family of selfadjoint linear operators on $L^{2}(S, d x)$, where $(S, d x)$ is a $\sigma$-finite measure space. Suppose that the following conditions hold:

(i) $T_{\mu}$ are uniformly bounded.

(ii) $T_{\mu} f(x) \geq 0$ if $f(x) \geq 0$ for all $\mu$.

(iii) If $f \geq 0$, then

$$
T_{\mu} T_{\nu} f(x) \leq M_{\mu} T_{\varphi(\nu)} f(x)+N_{\nu} T_{\varphi(\mu)} f(x),
$$

a.e. for all $\mu$ and $\nu$ where $\varphi$ is a fixed function from $A$ into $A$ and where $M_{\mu}, N_{\nu}$ are linear operators on $L^{2}(S)$ satisfying the following estimates

$$
\left\|\sup _{\mu} M_{\mu} f(x)\right\|_{L^{2}} \leq M\|f\|_{L^{2}}
$$

and

$$
\left\|\sup _{\mu} N_{\mu} f(x)\right\|_{L^{2}} \leq N\|f\|_{L^{2}}
$$

for some constants $M, N$.

(iv) For a dense subspace $X$ of $L^{2}(S)$, we have

$$
\left\|\sup _{\mu} T_{\mu} f(x)\right\|_{L^{2}} \text { is finite. }
$$

Then $\left\|\sup _{\mu} T_{\mu} f(x)\right\|_{L^{2}} \leq C\|f\|_{L^{2}}$, where $C$ can be taken to be $M+N$.

Proof. Write $A=\bigcup_{i=1}^{\infty} A_{i}$ where each $A_{i}$ is finite and for all $i, A_{i} \subset A_{i+1}$. We will first prove the proposition for the finite case. Let $m$ be a positive integer and let $\mu(x)$ be any measurable function with values on $A_{m}$. By condition (iii) we have that for any $f \geq 0$

$$
T_{\mu(x)} T_{\nu} f(x) \leq M_{\mu(x)} T_{\varphi(\nu)} f(x)+N_{\nu} T_{\varphi(\mu(x))} f(x) .
$$


Since the operators $T_{\mu}$ are selfadjoint and positive, by taking adjoints on equation (1.5), we obtain

$$
T_{\nu} T_{\mu(x)}^{*} f(x) \leq T_{\varphi(\nu)} M_{\mu(x)}^{*} f(x)+T_{\varphi(\mu(x))}^{*} N_{\nu}^{*} f(x),
$$

and since $f(x) \geq 0$, we take $\nu=\mu(x)$ and obtain that

(1.7) $\left(T_{\mu(x)}^{*} f, T_{\mu(x)}^{*} f\right) \leq\left(M_{\mu(x)}^{*} f(x), T_{\varphi(\mu(x))}^{*} f(x)\right)+\left(N_{\mu(x)}^{*} f(x), T_{\varphi(\mu(x))}^{*} f(x)\right)$.

Let $f \geq 0$ be fixed. Given $\varepsilon>0$, we choose $\mu(x)$ so that

$$
(1+\varepsilon) T_{\mu(x)} f(x)>\sup _{\mu \in A_{m}} T_{\mu} f(x) .
$$

Then by (1.7), (1.2) and (1.3) we have that if $\|f\|_{L^{2}} \leq 1$

$$
\left\|\sup _{\mu \in A_{m}} T_{\mu} f(x)\right\|_{L^{2}}^{2}<(1+\varepsilon)(M+N)\left\|\sup _{\mu \in A_{m}} T_{\varphi(\mu)} f(x)\right\|_{L^{2}}
$$

for all $f \in L^{2}$ with $\|f\|_{L^{2}} \leq 1$ since (1.9) is independent of $\mu(x)$. And, since $\varepsilon$ can be made arbitrarily small, we have that

$$
\left\|\sup _{\mu \in A_{m}} T_{\mu} f(x)\right\|_{L^{2}}^{2} \leq(M+N)\left\|\sup _{\mu \in A_{m}} T_{\varphi(\mu)} f(x)\right\|_{L^{2}} .
$$

If we assume further that $f \in X$, then by (iv)

$$
\left\|\sup _{\mu \in A_{m}} T_{\mu} f(x)\right\|_{L^{2}}^{2} \leq(M+N)\left\|\sup _{\mu} T_{\mu} f(x)\right\|_{L^{2}}<\infty
$$

for $f \in L^{2} \cap X$ and $\|f\|_{L^{2}} \leq 1$.

This proves the proposition for the finite case and $f \in L^{2} \cap X$. But the right-hand side of (1.11) is independent of $m$ so that if we let $m \rightarrow \infty$, we obtain

$$
\left\|\sup _{\mu} T_{\mu} f(x)\right\|_{L^{2}} \leq(M+N)
$$

for all $f \in X$ with $\|f\|_{L^{2}} \leq 1$. Since $X$ is dense in $L^{2}$ we have that (1.18) holds for all $f \in L^{2}$ with $\|f\|_{L^{2}} \leq 1$ which is the desired conclusion.

In the rest of the paper, we will be working on $\mathbf{R}^{n}$ with the usual Lebesgue measure.

\section{GEOMETRIC CONSIDERATIONS}

To estimate $T_{\mu} T_{\nu} f$, we will need to use some simple properties of convolutions in $\mathbf{R}^{n}$.

The following fact is well known, see [Hör, p. 102].

Proposition 2.1. Let $u$ and $v$ be distributions on $\mathbf{R}^{n}$ with compact support. Then $u * v$ is a distribution with compact support and

$$
\operatorname{supp}(u * v) \subset \operatorname{supp} u+\operatorname{supp} v=\{x+y: x \in \operatorname{supp} u, y \in \operatorname{supp} v\} .
$$

Definition. A set $R$ in $\mathbf{R}^{n}$ will be called a rectangle if it is congruent to $\left[a_{1}, b_{1}\right] \times \cdots \times\left[a_{n}, b_{n}\right]$ for some $a_{i}$ and $b_{i}$ with $a_{i}<b_{i}, 1 \leq i \leq n$. 
Definition. Let $\varepsilon$ be a fixed number satisfying $0<\varepsilon \leq 1$. We define $\mathscr{B}_{\varepsilon}$ to be the class of all rectangles in $\mathbf{R}^{n}$ that are symmetric about the origin and congruent to some dilate of $\left[-\frac{1}{2}, \frac{1}{2}\right]^{n-1} \times\left[-\frac{\varepsilon}{2}, \frac{\varepsilon}{2}\right]$. Similarly we define $\mathscr{E}_{\varepsilon}$ to be the class of all cylinders in $\mathbf{R}^{n}$ that are symmetric about the origin and congruent to some dilate of the cylinder

$$
\left\{x=\left(x_{1}, \ldots, x_{n}\right) \in \mathbf{R}^{n}: x_{1}^{2}+\cdots+x_{n-1}^{2} \leq \frac{1}{4} \text { and }\left|x_{n}\right|<\frac{\varepsilon}{2}\right\} .
$$

An element of $\mathscr{C}_{\varepsilon}$ is a cylinder $D$ centered at the origin with a base of radius $\frac{h}{2}$ and height $\varepsilon h$ and thus it can be described by the parameter $\mu=(h, \theta)$ where $\theta \in S^{n-1}$ is a normal vector to the base of the cylinder. This association will be one to one if we identify the points $(h, \theta)$ and $(h,-\theta)$ on $\mathbf{R}^{+} \times S^{n-1}$.

Thus, each $D \in \mathscr{C}_{\varepsilon}$ will be denoted by $D_{\mu}$.

For a given $\mu=(h, \theta)$, let $\mathscr{B}_{\varepsilon}^{\mu}$ denote the class of all rectangles in $\mathscr{B}_{\varepsilon}$ that are congruent to $\left[-\frac{h}{2}, \frac{h}{2}\right]^{n-1} \times\left[-\frac{\varepsilon h}{2}, \frac{\varepsilon h}{2}\right]$ and have its largest face perpendicular to $\theta$.

The following lemma states that, for our purposes, all the rectangles in $\mathscr{B}_{\varepsilon}^{\mu}$ and $D_{\mu} \in \mathscr{C}_{\varepsilon}$ are equivalent.

Lemma 2.2. Let $\mu=(h, \theta)$ and let $\mathscr{B}_{\varepsilon}^{\mu}$ be as above. Then for all $R \in \mathscr{B}_{\varepsilon}^{\mu}$ we have that for all $x \in \mathbf{R}^{n}$

$$
\frac{C_{1}}{\left|D_{\mu}\right|} \chi_{D_{\mu}}(x) \leq \frac{1}{|R|} \chi_{R}(x) \leq \frac{C_{2}}{\left|D_{\mu^{\prime}}\right|} \chi_{D_{\mu^{\prime}}}(x)
$$

where $\mu^{\prime}=(\sqrt{n-1} h, \theta)$ and $C_{1}$ and $C_{2}$ are constants depending only on the dimension $n$ and not on $\mu$ or $\varepsilon$.

The proof is simple and is left to the reader.

Now, let $\mu=(h, \theta), \nu=(\kappa, \gamma)$ and let $R_{1} \in \mathscr{B}_{\varepsilon}^{\mu}, R_{2} \in \mathscr{B}_{\varepsilon}{ }_{\varepsilon}$. Let $\varphi_{1}(x)$, $\varphi_{2}(x)$ be given by

$$
\varphi_{i}(x)=\frac{1}{\left|R_{i}\right|} \chi_{R_{i}}(x), \quad i=1,2 .
$$

To estimate $\varphi_{1} * \varphi_{2}(x)$, we have the following easy proposition that we prove for completeness.

Proposition 2.3. With the above notation, we have that for all $x \in \mathbf{R}^{n}$

$$
\varphi_{1} * \varphi_{2}(x) \leq C \frac{1}{|E|} \chi_{E}
$$

where $E$ is a rectangle in $\mathbf{R}^{n}$ symmetric about the origin congruent to $\left[-\frac{a}{2}, \frac{a}{2}\right]^{n-1}$ $\times\left[-\frac{b}{2}, \frac{b}{2}\right]$ with $a$ and $b$ satisfying

(2.3) $a=\max \left(2 h^{\prime}, 2 \kappa^{\prime}\right), \quad$ where $h^{\prime}=(n-1)^{1 / 2} h$ and $\kappa^{\prime}=(n-1)^{1 / 2} \kappa$,

(2.4) $b=\max (2 \varepsilon a, d \sin \hat{\theta} \gamma)$, where $d=\min \left(2 h^{\prime}, 2 \kappa^{\prime}\right)$.

$E$ is oriented in such a way that its largest face is normal to $\theta$ or $\gamma$ according to whether $h \geq \kappa$ or $\kappa>h$. $\hat{\theta} \gamma$ denotes the angle between $\theta$ and $\gamma$. The constant $C$ depends only on the dimension $n$ and not on the rectangles $R_{1}$ and $R_{2}$.

Proof. We want to estimate

$$
\varphi_{1} * \varphi_{2}(x)=\int_{\mathbf{R}^{n}} \varphi_{1}(x-y) \varphi_{2}(y) d y .
$$


By Lemma 2.2 we have that

$$
\begin{aligned}
\varphi_{1}(x) * \varphi_{2}(x) & \leq C \tilde{\varphi}_{1} * \tilde{\varphi}_{2}(x), \quad \text { where } \tilde{\varphi}_{1}(x)=\frac{1}{\left|D_{\mu^{\prime}}\right|} \chi_{D_{\mu^{\prime}}}(x), \\
\tilde{\varphi}_{2}(x) & =\frac{1}{\left|D_{\nu^{\prime}}\right|} \chi_{D_{\nu^{\prime}}}(x) \quad \text { with } \mu^{\prime}=(\sqrt{n-1} h, \theta)=\left(h^{\prime}, \theta\right), \\
\nu^{\prime} & =(\sqrt{n-1} \kappa, \gamma)=\left(\kappa^{\prime}, \gamma\right) .
\end{aligned}
$$

There is no loss of generality in assuming that $h \geq \kappa$. Then, by changing coordinates if necessary, we can also assume that $\theta=e_{n}$, that is

$$
D_{\mu^{\prime}}=\left\{\left(x_{1}, \ldots, x_{n}\right) \in \mathbf{R}^{n}: x_{1}^{2}+\cdots+x_{n-1}^{2} \leq h^{\prime 2} \text { and }\left|x_{n}\right| \leq \varepsilon h^{\prime} / 2\right\} .
$$

By applying Lemma 2.2 again, we have that

$$
\tilde{\varphi}_{1} * \tilde{\varphi}_{2}(x) \leq C \psi_{1} * \psi_{2}(x)
$$

where

$$
\psi_{1}(x)=\frac{1}{\left|\widetilde{R}_{1}\right|} \chi_{\widetilde{R}_{1}}(x), \quad \psi_{2}(x)=\frac{1}{\left|\widetilde{R}_{2}\right|} \chi_{\widetilde{R}_{2}}(x)
$$

where $\widetilde{R}_{1}$ is the rectangle $\left\{x \in \mathbf{R}^{n}:\left|x_{i}\right| \leq h^{\prime} / 2, i=1, \ldots, n-1\right.$, and $\left.\left|x_{n}\right|<\varepsilon h^{\prime} / 2\right\}$ and $\widetilde{R}_{2}$ is the rectangle $\left\{x \in \mathbf{R}^{n}:\left|x_{i}\right|<\kappa^{\prime} / 2, i=1, \ldots, n-1\right.$, and $\left.\left|x_{n}\right|<\varepsilon \kappa^{\prime} / 2\right\}$ rotated by an angle of $\widehat{\theta \gamma}$ about the $e_{2}, \ldots, e_{n-1}$-plane, that is

$$
\begin{aligned}
& \widetilde{R}_{2}=\left\{x \in \mathbf{R}^{n}:\left|x_{i}\right| \leq \kappa^{\prime} / 2 \text { for } i=2,3, \ldots, n-1 ;\right. \\
& \left.\quad\left|x_{1} \cos \widehat{\theta \gamma}+x_{n} \sin \widehat{\theta \gamma}\right| \leq \kappa^{\prime} / 2 \text { and }\left|x_{n} \cos \widehat{\theta \gamma}-x_{1} \sin \widehat{\theta \gamma}\right| \leq \varepsilon \kappa^{\prime} / 2\right\} .
\end{aligned}
$$

In order to estimate $\psi_{1} * \psi_{2}(x)$, we will use the following formula that can be easily obtained

$$
\psi_{1} * \psi_{2}(x)=\int_{\mathbf{R}^{n}} \psi_{1}(y) \psi_{2}(x-y) d y=\frac{\left|\widetilde{R}_{1} \cap\left(\widetilde{R}_{2}+x\right)\right|}{\left|\widetilde{R}_{1}\right|\left|\widetilde{R}_{2}\right|}
$$

where $\left|\widetilde{R}_{1}\right|=\left(h^{\prime}\right)^{n} \varepsilon$ and $\left|\widetilde{R}_{2}\right|=\left(\kappa^{\prime}\right)^{n} \varepsilon$.

There are two cases:

Case (i). $2 \varepsilon a \geq d \sin \widehat{\theta \gamma}$ : in this case since we assumed $h>\kappa$, we have $4 \varepsilon h^{\prime} \geq 2 \kappa^{\prime} \sin \widehat{\theta \gamma}$. By Proposition 2.1 we have that $\psi_{1} * \psi_{2}$ will have support contained in the rectangle $E=\left\{x:\left|x_{i}\right| \leq h^{\prime}\right.$ for $\left.i \leq n-1,\left|x_{n}\right| \leq 2 \varepsilon h^{\prime}\right\}$ and thus $|E|=2^{n+1}\left(h^{\prime}\right)^{n} \varepsilon$. It is clear from (2.9) that for all $x$ in $E$ we have

$$
\psi_{1} * \psi_{2}(x) \leq \frac{1}{\left|\widetilde{R}_{1}\right|}=\frac{1}{\left(h^{\prime}\right)^{n} \varepsilon} .
$$

Thus

$$
\psi_{1} * \psi_{2}(x) \leq 2^{n+1} \frac{1}{|E|} \chi_{E}(x)
$$

Case (ii). $d \sin \widehat{\theta \gamma}>2 \varepsilon a$, that is $2 \kappa^{\prime} \sin \widehat{\theta \gamma}>4 \varepsilon h^{\prime}$. 
By Proposition 2.1, $\psi_{1} * \psi_{2}$ is supported on the rectangle $E=\left\{x \in \mathbf{R}^{n}\right.$ : $\left|x_{i}\right| \leq h^{\prime}$ for $\left.i \leq n-1,\left|x_{n}\right| \leq \kappa^{\prime} \sin \widehat{\theta \gamma}\right\}$ and $|E|=2^{n+1}\left(h^{\prime}\right)^{n-1} \kappa^{\prime} \sin \widehat{\theta \gamma}$. By (2.9) we have that

$$
\psi_{1} * \psi_{2}(0)=\frac{\kappa^{n-2}\left(\varepsilon h^{\prime}\right)\left(\varepsilon \kappa^{\prime} / \sin \widehat{\theta \gamma}\right)}{\left[\left(h^{\prime}\right)^{n} \varepsilon\right]\left[\left(k^{\prime}\right)^{n} \varepsilon\right]}=\frac{1}{\left(h^{\prime}\right)^{n-1} \kappa^{\prime} \sin \widehat{\theta \gamma}}=\frac{2^{n+1}}{|E|} .
$$

And by translating $\widetilde{R}_{2}$, it is clear that the measure of $\left|\widetilde{R}_{1} \cap\left(x+\widetilde{R}_{2}\right)\right|$ cannot increase so that we have

$$
\psi_{1} * \psi_{2}(x) \leq \frac{2^{n+1}}{|E|} \quad \text { for all } x \text { in } E
$$

Thus

$$
\psi_{1} * \psi_{2}(x) \leq 2^{n+1} \frac{1}{|E|} \chi_{E}(x) \quad \text { for all } x \in \mathbf{R}^{n} .
$$

By (2.6), (2.7), (2.10) and (2.13), we have that

$$
\varphi_{1} * \varphi_{2}(x) \leq C \frac{1}{|E|} \chi_{E}(x) \quad \text { for all } x \in \mathbf{R}^{n}
$$

where $C$ is a constant depending only on the dimension $n$ and $E$ is the rectangle described in the statement of the proposition.

A similar result holds for some more singular measures on $\mathbf{R}^{2}$ : for $h>0$ and $\theta \in\left[\frac{-\pi}{4}, \frac{\pi}{4}\right]$, let $u_{(h, \theta)}$ be the distribution whose action on test functions is given by

$$
u_{(h, \theta)}(f)=\frac{1}{2 h} \int_{-h}^{h} f(t \cos \theta, t \sin \theta) d t, \quad f \in C_{0}^{\infty}\left(\mathbf{R}^{2}\right) .
$$

Then we have

Proposition 2.4. If $f \in C_{0}^{\infty}\left(\mathbf{R}^{2}\right)$ is nonnegative, then for all $(x, y) \in \mathbf{R}^{2}$, we have that

(i) $u_{(h, \theta)} * u_{(\kappa, \theta)} * f(x, y) \leq 2 u_{(2 \max (h, \kappa), \theta)} * f(x, y)$, or

(ii) $u_{(h, \theta)} * u_{(\kappa, \gamma)} * f(x, y) \leq(4 /|E|) \chi_{E} * f(x, y)$

where $E$ is a rectangle of dimensions $4 \max (h, \kappa)$ and $2 \min (h, \kappa) \sin |\theta-\gamma|$, symmetric about the origin and with its longest side parallel to $\theta$ or $\gamma$ according to whether $h>\kappa$ or $h \leq \kappa$.

The proof is similar to the preceding one and is left to the reader.

Remark. Since for any rectangle $E$ as above, there exists a parallelogram $P$ centered at the origin, containing $E$ such that

(a) its longest sides are parallel to the longest sides of $E$;

(b) the shortest sides of $P$ are parallel to one of the coordinate axis;

(c) $|P| \leq 2|E|$,

we obtained that the conclusion (ii) above can be replaced by

$$
u_{(h, \theta)} * u_{(\kappa, \gamma)} * f(x, y) \leq \frac{8}{|P|} \chi_{P} * f(x, y) .
$$




\section{Proof of THEOREMS}

Before we proceed in proving the theorems, we will make some simplifications in order to apply the results of the preceding paragraphs.

Since $M_{N} f=M_{N}|f|$, it is enough to prove Theorem A for nonnegative functions.

For $N>2$ fixed, let $m$ be an integer such that $2^{m} \leq N<2^{m+1}$. Then if $R$ is a rectangle in $\mathbf{R}^{n}$ containing $x$ congruent to $[0, h]^{h-1} \times\left[0, h N^{-1}\right]$ where $h>0$ satisfies $2^{i}<h<2^{i+1}$ for some $i \in \mathbf{Z}$, we have that for all $f \geq 0$

$$
\frac{1}{|R|} \int_{R}|f(y)| d y \leq C_{n} f * \frac{1}{\left|R^{\prime}\right|} \chi_{R^{\prime}}(x)
$$

where $R^{\prime}$ is a rectangle centered at the origin, congruent to $\left[-2^{i}, 2^{i}\right]^{n-1} \times$ $\left[-2^{i-m}, 2^{i-m}\right]$ and with sides parallel to $R$, that is, $R^{\prime}$ belongs to $\mathscr{B}_{2^{-m}}$ and has the same orientation as $R$. Thus $R^{\prime}=R_{\mu}$ with $\mu=\left(2^{i}, \theta\right), i \in \mathbf{Z}$, $\theta \in S^{n-1}$. Also, by continuity of the Lebesgue integral, we can assume that $\theta$ belongs to a dense countable subset $\Omega$ of $S^{n-1}$.

Thus, Theorem A is equivalent to

Theorem $\mathbf{A}^{\prime}$. Let $\Omega=\left\{w_{j}\right\}_{j \in Z}$ be a dense countable subset of $S^{n-1}$. For $\mu=(i, j) \in \mathbf{Z}^{2}$, let $R_{\mu}$ be the rectangle in $\mathscr{B}_{2^{-m}}$ congruent to $\left[-2^{i}, 2^{i}\right] \times$ $\left[-2^{i-m}, 2^{i-m}\right]$ with $w_{j}$ as a normal to its largest face. Define $T_{\mu}^{m}$ and $T^{m}$ by

$$
\begin{aligned}
T_{\mu}^{m} f(x) & =\frac{1}{\left|R_{\mu}\right|} \chi_{R_{\mu}} * f(x), \\
T^{m} f(x) & =\sup _{\mu}\left|T_{\mu}^{m} f(x)\right| .
\end{aligned}
$$

Then

$$
\left\|T^{m} f\right\|_{L^{2}\left(\mathbf{R}^{n}\right)} \leq C_{n} m\|f\|_{L^{2}\left(\mathbf{R}^{n}\right)} .
$$

Proof. By positivity, we only need to prove (3.4) for $f \geq 0$.

Clearly $T_{\mu}^{m}$ is a bounded selfadjoint operator on $L^{2}$ and if $f(x) \geq 0$ a.e. then $T_{\mu}^{m} f(x) \geq 0$ everywhere for all $\mu$.

In order to apply the ideas of $\S 1$, we need to estimate $T_{\mu}^{m} T_{\nu}^{m} f(x)$ for $f \geq 0$. Let $\mu=(i, j), \nu=(k, l)$, then by Proposition 2.3, we have that

$$
T_{\mu}^{m} T_{\nu}^{m} f(x) \leq C_{1} \frac{1}{|E|} \chi_{E} * f(x)
$$

where $C_{1}$ depends only on the dimension $n$ and $E$ is a rectangle in $\mathbf{R}^{n}$ symmetric about the origin, congruent to $\left[-\frac{a}{2}, \frac{a}{2}\right]^{n-1} \times\left[-\frac{b}{2}, \frac{b}{2}\right]$ with $a$ and $b$ satisfying (2.3) and (2.4) and $E$ is oriented according to the statement of Proposition 2.3. Thus $E$ is an element of some $\mathscr{B}_{2-s}$ where $s$ satisfies $1 \leq s \leq m-1$ and $E$ is parallel either to $R_{\mu}$ or $R_{\nu}$ according to whether $i \leq k$ or $k<i$. Thus if $\varphi: \mathbf{Z}^{2} \rightarrow \mathbf{Z}^{2}$ is defined by $\varphi((i, j))=\left(i^{\prime}, j\right)$ where $i^{\prime}$ satisfies $2^{i^{\prime}-1}<(n-1)^{1 / 2} 2^{i} \leq 2^{i^{i}}$, we have that

$$
T_{\mu}^{m} T_{\nu}^{m} f(x) \leq C_{1}\left(T_{\varphi(\mu)}^{s} f(x)+T_{\varphi(\nu)}^{s} f(x)\right)
$$

where $s=s(\mu, \nu) \leq m-1$. We still have to eliminate the dependence of $s$ on $\mu$ and $\nu$ and this is done by summing in $s$. One has

$$
T_{\mu}^{m} T_{\nu}^{m} f(x) \leq C_{1} \sum_{s=1}^{m-1} T_{\varphi(\mu)}^{s} f(x)+T_{\varphi(\nu)}^{s} f(x) .
$$


By repeating the steps on the proof of Proposition 1.1, we obtain

$$
\left\|T^{m} f\right\|_{L^{2}\left(\mathbf{R}^{n}\right)}^{2} \leq 2 C_{1} \sum_{s=1}^{m-1}\left\|T^{s} f\right\|_{L^{2}\left(\mathbf{R}^{n}\right)} .
$$

The proof is now completed by induction on $m$ :

$m=1: T^{1}$ is dominated by the Hardy-Littlewood maximal operator, so $\left\|T^{1} f\right\|_{L^{2}} \leq C\|f\|_{L^{2}}$. Suppose that for all $s<m$ we have that $\left\|T^{s} f\right\|_{L^{2}} \leq$ $C s\|f\|_{L^{2}}$ where $C$ is independent of $s$ and $f$. We can assume that $C>C_{1}$. Then by (3.8)

$$
\left\|T^{m} f\right\|_{L^{2}}^{2} \leq 2 C^{2} \sum_{s=1}^{m-1} s\|f\|_{L^{2}} \leq C^{2} m^{2}\|f\|_{L^{2}} .
$$

This completes the proof of Theorem $\mathrm{A}^{\prime}$ and hence of Theorem A.

As a corollary we immediately obtain an improved version of Theorem 1 in [Cor 1]. This was previously obtained by Strömberg in [Str 1] by covering lemma arguments.

Corollary 3.1. Let

$$
M_{N} f(x)=\sup _{x \in R} \frac{1}{|R|} \int_{R}|f(y)| d y,
$$

where the sup is taken over all rectangles in $\mathbf{R}^{2}$ satisfying

$$
\frac{\text { largest side of } R}{\text { shortest side of } R}=N \text {. }
$$

Then for all $f \in L^{2}\left(\mathbf{R}^{2}\right)$

$$
\left\|M_{N} f\right\|_{L^{2}\left(\mathbf{R}^{2}\right)} \leq C(\log N)\|f\|_{L^{2}\left(\mathbf{R}^{2}\right)} .
$$

Proof. This is just the case $n=2$ of Theorem A.

To prove Theorem $B$, we first have to introduce some more notation. As in the statement of Theorem A, let $\Omega=\left\{w_{j}\right\}_{j \in \mathbf{Z}}$ be a countable dense subset of $S^{n-1}$. In order to simplify the argument, we will assume that for each $w_{j} \in \Omega$, there exists a $w_{k}$ in $\Omega$ such that $w_{k}$ is orthogonal to $w_{j}$. Let $m$ be a positive integer. For $\mu=(i, j) \in \mathbf{Z}^{2}$, let $C_{\mu}$ be the cylinder, symmetric about the origin, congruent to $\left\{x=\left(x_{1}, \ldots, x_{n}\right):-2^{i-1} \leq x_{1} \leq 2^{i-1},\left(x_{2}^{2}+\cdots+x_{n}^{2}\right)^{1 / 2}<2^{i-m}\right\}$ and such that its axis is parallel to $w_{j}$.

Define $S_{\mu}^{m} f(x)$ by

$$
S_{\mu}^{m} f(x)=\frac{1}{\left|C_{\mu}\right|} \chi_{C_{\mu}} * f(x) .
$$

An argument similar to the one preceding Theorem $\mathrm{A}^{\prime}$ shows that Theorem $B$ is equivalent to the following.

Theorem $\mathbf{B}^{\prime}$. With the above notation, let

$$
S^{m} f(x)=\sup _{\mu}\left|S_{\mu}^{m} f(x)\right| .
$$

Then for all $f \in L^{2}\left(\mathbf{R}^{n}\right)$, we have

$$
\left\|S^{m} f\right\|_{L^{2}\left(\mathbf{R}^{n}\right)} \leq C 2^{m\left(\frac{n-2}{2}\right)} m\|f\|_{L^{2}\left(\mathbf{R}^{n}\right)} .
$$




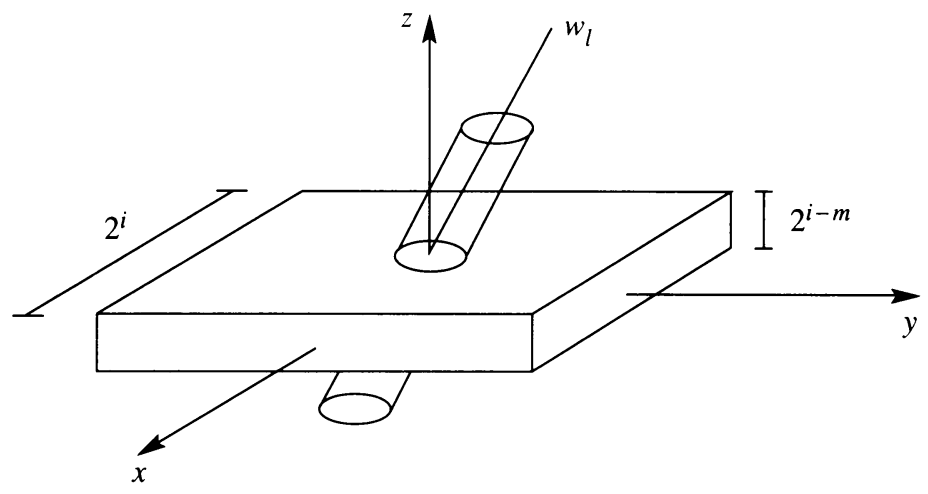

FiguRE

Proof. For $\mu=(i, j)$, let $\varphi(\mu)=(i, k(j))$ where $w_{j}$ is orthogonal to $w_{k}$. Thus, if $T_{\mu}^{m} f$ is defined as in Theorem $\mathrm{A}^{\prime}$, we have that for all $f \geq 0$.

$$
S_{\mu}^{m} f(x) \leq 2^{m(n-2)} T_{\varphi(\mu)}^{m} f(x) \quad \text { a.e. for all } \mu \in \mathbf{Z}^{2} .
$$

The theorem is then a consequence of the following.

Lemma 3.2. For all $\mu, \nu \in \mathbf{Z}^{2}$ and $f \geq 0$ we have

$$
S_{\mu}^{m} S_{\nu}^{m} f(x) \leq C 2^{m(n-2)} \sum_{s=1}^{m-1} T_{\beta(\varphi(\mu))}^{s} f(x)+T_{\beta(\varphi(\nu))}^{s} f(x)
$$

where $C$ is a constant depending only on the dimension, $\beta: \mathbf{Z}^{2} \rightarrow \mathbf{Z}^{2}$ is a fixed function and $\varphi$ is the function on $\mathbf{Z}^{2}$ described above.

Thus applying the reasoning of Proposition 1.1, we obtain from (3.16) that

$$
\left\|S^{m} f\right\|_{L^{2}\left(\mathbf{R}^{n}\right)}^{2} \leq C 2^{m(n-2)} \sum_{s=1}^{m-1}\left\|T^{s} f\right\|_{L^{2}\left(\mathbf{R}^{n}\right)} .
$$

By Theorem $\mathrm{A}^{\prime},\left\|T^{s} f\right\|_{L^{2}\left(\mathbf{R}^{n}\right)} \leq C s$ for all $f \in L^{2}\left(\mathbf{R}^{n}\right)$ satisfying $\|f\|_{L^{2}\left(\mathbf{R}^{n}\right)} \leq$ 1. Thus we have

$$
\left\|S^{m} f\right\|_{L^{2}\left(\mathbf{R}^{n}\right)}^{2} \leq C 2^{m(n-2)} m^{2} \text { for all } f \text { with }\|f\|_{L^{2}\left(\mathbf{R}^{n}\right)} \leq 1 .
$$

In order to complete the proof, we just need to prove Lemma 3.2.

Proof of Lemma 3.2. The proof is a combination of the ideas in Proposition 2.3 and Theorem $\mathrm{A}^{\prime}$. Let $\mu=(i, j), \nu=(k, l)$ and assume that $i>k$. By (3.15) we have that for $f \geq 0$

$$
S_{\mu}^{m} S_{\nu}^{m} f(x) \leq 2^{m(n-2)} T_{\varphi(\mu)}^{m} S_{\nu}^{m} f(x)
$$

where

$$
T_{\varphi(\mu)}^{m} f(x)=\frac{1}{\left|R_{\mu}\right|} \chi_{R_{\mu}} * f(x) \quad \text { and } \quad S_{\nu}^{m} f(x)=\frac{1}{\left|C_{\nu}\right|} \chi_{C_{\nu}} * f(x)
$$

and after changing coordinates, if necessary we can assume that $R_{\mu}=\left\{x \in \mathbf{R}^{n}\right.$ : $\left|x_{p}\right|<2^{i-1}$ for $p \leq n-1$ and $\left.\left|x_{n}\right|<2^{i-1-m}\right\}$. See the figure for $n=3$. 
An argument similar to the one in Proposition 2.3 shows that

$$
T_{\varphi(\mu)}^{m} S_{\nu}^{m} f(x) \leq \frac{C}{|E|} \chi_{E} * f(x)
$$

where $E$ is the rectangle $\left\{x \in \mathbf{R}^{n}:\left|x_{p}\right| \leq 2^{i}\right.$ for $\left.p \leq n-1,\left|x_{n}\right| \leq d\right\}$ where $d=\max \left(2^{i-m+1}, 2^{k} \cos \widehat{w_{l} e_{n}}\right)$. Thus if $\beta(i, j)=(2 i, j)$ we get

$$
T_{\varphi(\mu)}^{m} S_{\nu}^{m} f(x) \leq C T_{\beta(\varphi(\mu))}^{s(\mu, \nu)} f(x), \quad \text { where } s(\mu, \nu) \leq m-1 .
$$

As in Theorem $\mathrm{A}^{\prime}$, we add in $s$ and get

$$
S_{\mu}^{m} S_{\nu}^{m} f(x) \leq C 2^{m(n-2)} \sum_{s=1}^{m-1} T_{\beta(\varphi(\mu))}^{s} f(x) \quad \text { if } i>k .
$$

The case $i<k$ is obtained by "interchanging" $\mu$ and $\nu$. Note that (3.16) includes both cases. This finishes the proof of the lemma and of the theorem.

We will now show by examples that the power of $\log N$ in Theorem A and the power of $N$ in Theorem B cannot be improved.

Example A. For $N \gg 1, x=\left(x_{1}, \ldots, x_{n}\right)$ in $\mathbf{R}^{n}$, let $C_{N}$ be the "cylinder" $\left\{x \in \mathbf{R}^{n}: 1 \leq x_{1}^{2}+x_{2}^{2} \leq N^{2}, 0 \leq x_{i} \leq N\right.$ for $\left.2<i \leq n\right\}$.

Let

then

$$
f_{N}(x)=\frac{1}{\left(x_{1}^{2}+x_{2}^{2}\right)^{1 / 2}} \chi_{C_{N}}(x)
$$

$$
\left\|f_{N}\right\|_{L^{2}}=C N^{(n-2) / 2}(\log N)^{1 / 2} .
$$

If $x$ satisfies $x_{1}^{2}+x_{2}^{2} \sim j^{2}$, an easy computation shows that

$$
M_{N} f_{N}(x) \geq C j^{-1} \log j
$$

and thus

$$
\left\|M_{N} f_{N}\right\|_{L^{2}\left(\mathbf{R}^{n}\right)}^{2} \geq C \sum_{j=1}^{N} N^{n-2} \frac{(\log j)^{2}}{j} \sim N^{n-2}(\log N)^{3} .
$$

Combining (3.24) and (3.26), we obtain that

$$
\left\|M_{N} f_{N}\right\|_{L^{2}\left(\mathbf{R}^{n}\right)} \geq C(\log N)\left\|f_{N}\right\|_{L^{2}\left(\mathbf{R}^{n}\right)} .
$$

Example B. Let $f$ be the characteristic function of the unit ball in $\mathbf{R}^{n}$ so that $\|f\|_{L^{2}\left(\mathbf{R}^{n}\right)}=C_{n}$.

For $x$ in $\mathbf{R}^{n}$ satisfying $|x|>N$, it is easy to see that

$$
\widetilde{M}_{N} f(x)=C N^{(n-1)}|x|^{-n}
$$

and thus

$$
\left\|\widetilde{M}_{N} f\right\|_{L^{2}} \geq C N^{(n-2) / 2}
$$

The following maximal operator has been considered by Cordoba in [Cor 3]. Let $\left\{w_{j 0}\right\}_{j=1}^{\infty}$ be a lacunary sequence converging to zero, that is, there exists a number $\lambda$ satisfying $0<\lambda<1$ and such that

$$
\frac{w_{j+1,0}}{w_{j, 0}} \leq \lambda, \quad w_{1,0}=1
$$


Let $N$ satisfy $N \gg 1$, and for $k=0,1, \ldots, N-1$, let $w_{j k}$ satisfy

$$
w_{j+1,0}<w_{j k} \leq w_{j, 0}
$$

and

$$
\frac{a \Delta_{j}}{N}\left|k_{1}-k_{2}\right| \leq\left|w_{j k_{1}}-w_{j k_{2}}\right| \leq \frac{\Delta_{j}}{N}\left|k_{1}-k_{2}\right|
$$

where $\Delta_{j}=w_{j, 0}-w_{j+1,0}$ and $a$ satisfies $0<a<1$.

For locally integrable $f$ in $\mathbf{R}^{2}$ consider the maximal operator

$$
\mathscr{M}_{N} f(x)=\sup _{x \in R} \frac{1}{|R|} \int_{R}|f(y)| d y
$$

where the sup is taken over all rectangles in $\mathbf{R}^{2}$ having largest side parallel to $\left(1, w_{j k}\right)$ for some $j \geq 1$ and $k \leq N-1$.

Then it is proved in [Cor 3] that $\mathscr{M}_{N}$ satisfies the following weak-type estimate

$$
\left|\left\{x: \mathscr{M}_{N} f(x)>\alpha>0\right\}\right| \leq C_{\lambda, a}(\log 3 N) \alpha^{-2}\|f\|_{L^{2}\left(\mathbf{R}^{2}\right)}^{2}
$$

where $C_{\lambda, a}$ depends on $\lambda$ and $a$ but not on $N$ or $f$. Theorem C, stated in the introduction, shows that $\mathscr{M}_{N}$ is bounded on $L^{2}\left(\mathbf{R}^{2}\right)$, with the same norm essentially.

There is no loss in generality in assuming that the rectangles involved in (3.33) are centered at $x$ and have dimensions $2^{m_{1}} \times 2^{m_{2}}$ where $m_{1}, m_{2} \in \mathbf{Z}$. These affect $\mathscr{M}$ only by a multiplicative constant.

If $R_{0}$ is such a rectangle, with dimensions $2^{m_{1}} \times 2^{m_{2}} \quad\left(m_{1} \geq m_{2}\right)$ and with its largest side parallel to $\left(1, w_{j_{0} k_{0}}\right)$, then we have that for $f \geq 0$

$$
\frac{1}{\left|R_{0}\right|} \int_{R_{0}}|f(y)| d y \leq 8 S_{m_{2}} T_{\left(m_{1}, j_{0}, k_{0}\right)} f(x)
$$

where for $x=(x, y)$ in $\mathbf{R}^{2}$ we have

$$
S_{m} f(x, y)=\frac{1}{2^{m+1}} \int_{-2^{m}}^{2^{m}} f(x, y-s) d s
$$

and

$$
T_{(i, j, k)} f(x, y)=\frac{1}{2^{i+1}} \int_{-2^{i}}^{2^{i}} f\left(x-s, y-s w_{j k}\right) d s .
$$

Since $S_{m}$ is dominated by the one-dimensional Hardy-Littlewood maximal operator acting in the $y$-variable which is bounded on $L^{p}$ for $p>1$, Theorem C is then a consequence of

Theorem $\mathbf{C}^{\prime}$. For $i \in \mathbf{Z}, j=1,2, \ldots$, and $0 \leq k \leq N-1$, let $\mu=(i, j, k)$ and define $T_{\mu} f$ by

$$
T_{\mu} f(x, y)=\frac{1}{2^{i+1}} \int_{-2^{i}}^{2^{i}} f\left(x-s, y-s w_{j k}\right) d s,
$$

and

$$
T f(x, y)=\sup _{\mu}\left|T_{\mu} f(x, y)\right|
$$


then

$$
\|T f\|_{L^{2}\left(\mathbf{R}^{2}\right)} \leq C_{\lambda, a}(\log N)\|f\|_{L^{2}\left(\mathbf{R}^{2}\right)}
$$

Proof. Again, it suffices to prove (3.40) for $f \geq 0$. Let $\mu=\left(i_{1}, j_{1}, k_{1}\right)$, $\nu=\left(i_{2}, j_{2}, k_{2}\right)$ and let us estimate $T_{\mu} T_{\nu} f(x, y)$. These estimates will fall into two different cases.

Case 1. $\left|j_{1}-j_{2}\right| \geq 2$.

Assume, for the moment, that $j_{2}>j_{1}$. Then we have two subcases.

Subcase 1.1. $i_{2} \geq i_{1}$. By Proposition 2.4

$$
T_{\mu} T_{\nu} f(x, y) \leq \frac{8}{|E|} \chi_{E} * f(x, y)
$$

where $E$ is a parallelogram with longest sides parallel to $\left(1, w_{j_{2} k_{2}}\right)$ and length $2^{i_{2}+1}$ and vertical sides of length $2 d=2^{i_{1}+1}\left(w_{j_{1} k_{1}}-w_{j_{2} k_{2}}\right)$.

Since $w_{j_{1} k_{1}} /\left(w_{j_{1} k_{1}}-w_{j_{2} k_{2}}\right) \leq 1 /(1-\lambda)$ we have

$$
T_{\mu} T_{\nu} f(x, y) \leq \frac{8}{1-\lambda} A_{\mu} T_{\varphi(\nu)} f(x, y)
$$

where $\varphi(i, j, k)=(i+1, j, k)$ and

$$
A_{(i, j, k)} f(x, y)=\frac{1}{2^{i+1} w_{j k}} \int_{-2^{i} w_{j k}}^{2^{i} w_{j k}} f(x, y-s) d s .
$$

Subcase 1.2. $i_{1}>i_{2}$.

In this case, and inequality like (3.41) still holds with $E$ being a parallelogram with longest side parallel to $\left(1, w_{j_{1} k_{1}}\right)$ and horizontal sides of length $2 d$ where

$$
d=2^{i_{2}} \cdot \frac{\left(w_{j_{1} k_{1}}-w_{j_{1} k_{2}}\right)}{w_{j_{1} k_{1}}}
$$

By (3.30) we have that $d$ satisfies $2^{i_{2}}(1-\lambda) \leq d \leq 2^{i_{2}}$ so that

$$
T_{\mu} T_{\nu} f(x, y) \leq \frac{8}{1-\lambda} B_{\nu} T_{\varphi(\mu)} f(x, y)
$$

where

$$
B_{(i, j, k)} f(x, y)=\frac{1}{2^{i+1}} \int_{-2^{i}}^{2^{i}} f(x-s, y) d s .
$$
$\geq 2$

The case where $j_{1} \geq j_{2}$ is treated similarly and so we obtain that if $\left|j_{1}-j_{2}\right|$

Case 2. $\left|j_{1}-j_{2}\right| \leq 1$.

$$
\begin{aligned}
T_{\mu} T_{\nu} f(x, y) \leq \frac{C}{1-\lambda}\left(A_{\mu} T_{\varphi(\nu)} f(\right. & x, y)+A_{\nu} T_{\varphi(\mu)} f(x, y) \\
& \left.+B_{\mu} T_{\varphi(\nu)} f(x, y)+B_{\nu} T_{\varphi(\mu)} f(x, y)\right) .
\end{aligned}
$$

We again will first assume that $j_{2} \geq j_{1}$ and divide Case 2 into two subcases. Subcase 2.1. $i_{1} \geq i_{1}$.

Similarly to Subcase 1.1 , by Proposition 2.4 we have that an estimate like (3.41) holds where $E$ is a parallelogram with longest side parallel to $\left(1, w_{j_{2} k_{2}}\right)$ and length $2^{i_{2}+1}$ and vertical sides of length $2 d$ where $d$ satisfies

$$
\frac{2^{i_{1}} a(1-\lambda) w_{j_{1} 0}}{N} \leq d \leq 2^{i_{1}} w_{j_{1} 0},
$$

by (3.31) and (3.32). 
Now if $[z]$ denotes the greatest integer less or equal than $z$, let $L_{N}$ be defined by

$$
L_{N}=\left[\log \left(\frac{N}{a(1-\lambda)}\right) / \log 2\right]+1
$$

and for $l=0,1, \ldots, L_{N}$ let

$$
C_{(i, j, k)}^{l} f(x, y)=\left(\frac{2^{i+l+1}(1-\lambda) a w_{j 0}}{N}\right)^{-1} \int_{-2^{i+l}(1-\lambda) a w_{j 0} N^{-1}}^{2^{i+l}(1-\lambda) a w_{j 0} N^{-1}} f(x, y-s) d s .
$$

Then, as in the proof of Theorem $\mathrm{A}^{\prime}$, one has

$$
T_{\mu} T_{\nu} f(x, y) \leq 8\left(T_{\varphi(\mu)} f(x, y)+T_{\varphi(\nu)} f(x, y)+\sum_{l=0}^{L_{N}} C_{\mu}^{l} T_{\varphi(\nu)} f(x, y)\right) .
$$

Subcase 2.2. $i_{1}>i_{2}$.

As in Subcase 1.2, an estimate like (3.41) still holds only with the difference that now $d$ satisfies

$$
2^{i_{2}} a(1-\lambda) / N \leq d \leq 2^{i_{2}} .
$$

With $L_{N}$ as above, let

$$
D_{(i, j, k)}^{l} f(x, y)=\left(\frac{2^{i+l+1}(1-\lambda) a}{N}\right)^{-1} \int_{-2^{i+l}(1-\lambda) a N^{-1}}^{2^{i+l}(1-\lambda) a N^{-1}} f(x-s, y) d s .
$$

We then obtain

$$
T_{\mu} T_{\nu} f(x, y) \leq 8\left(T_{\varphi(\mu)} f(x, y)+T_{\varphi(\nu)} f(x, y)+\sum_{l=0}^{L_{N}} D_{\nu}^{l} T_{\varphi(\mu)} f(x, y)\right) .
$$

Similarly when $j_{1}>j_{2}$. By (3.46) and (3.53) we obtain that for all $\mu$ and $\nu$

$$
\begin{aligned}
T_{\mu} T_{\nu} f(x, y) \leq C_{\lambda}\left(T_{\varphi(\mu)}\right. & +T_{\varphi(\nu)}+A_{\mu} T_{\varphi(\nu)}+A_{\nu} T_{\varphi(\mu)} \\
& +B_{\mu} T_{\varphi(\nu)}+B_{\nu} T_{\varphi(\mu)}+\sum_{l=0}^{L_{N}} C_{\mu}^{l} T_{\varphi(\nu)} \\
& \left.+C_{\nu}^{l} T_{\varphi(\mu)}+D_{\mu}^{l} T_{\varphi(\nu)}+D_{\nu}^{l} T_{\varphi(\mu)}\right) f(x, y)
\end{aligned}
$$

where $C_{\lambda}=C /(1-\lambda)$.

Since operators $A_{\mu}, B_{\mu}, C_{\mu}^{l}$ and $D_{\mu}^{l}$ are dominated by a one-dimensional Hardy-Littlewood maximal operator acting in the $x$ or $y$ directions, we obtain

$$
\|T f\|_{L^{2}\left(\mathbf{R}^{2}\right)} \leq \frac{C}{1-\lambda}\left(1+L_{N}\right)\|f\|_{L^{2}\left(\mathbf{R}^{2}\right)}
$$

where $L_{N}$ is given by (3.48). Or equivalently

$$
\|T f\|_{L^{2}\left(\mathbf{R}^{2}\right)} \leq C_{\lambda, a}(\log N)\|f\|_{L^{2}\left(\mathbf{R}^{2}\right)}
$$

with $C_{\lambda, a}$ independent of $N$ and $f$.

This completes the proof of Theorem $\mathrm{C}^{\prime}$. 
The proof of Theorem $C^{\prime}$ contains as consequences $L^{2}$ versions of results previously obtained by different methods. See [Str 1, Str 2 and NSW].

Corollary 3.2. Let $\left\{w_{n}\right\}_{n=1}^{\infty}$ be a lacunary sequence converging to zero. Let

$$
\mathscr{M}_{1} f(x)=\sup _{x \in R} \frac{1}{|R|} \int_{R}|f(y)| d y,
$$

where the sup is taken over all rectangles in $\mathbf{R}^{2}$ with its longest side parallel to $\left(1, w_{n}\right)$ for some $n$. Then

$$
\left\|\mathscr{M}_{1} f\right\|_{L^{2}\left(\mathbf{R}^{2}\right)} \leq C_{\lambda}\|f\|_{L^{2}\left(\mathbf{R}^{2}\right)} .
$$

The proof of (3.58) is just a repetition of Case 1 in the proof of Theorem $\mathrm{C}^{\prime}$. This is also proved in [S1] using the same method.

Corollary 3.3. Let $e_{1}, e_{2}, \ldots, e_{N}$ be $N$ uniformly distributed directions in $\mathbf{R}^{2}$ and let

$$
\mathscr{M}^{N} f(x)=\sup _{x \in R} \frac{1}{|R|} \int_{R}|f(y)| d y
$$

where $R$ is any rectangle with one side parallel to the one of the $e_{j}$ 's. Then

$$
\left\|\mathscr{M}^{N} f\right\|_{L^{2}\left(\mathbf{R}^{2}\right)} \leq C(\log N)\|f\|_{L^{2}\left(\mathbf{R}^{2}\right)} .
$$

The proof of the corollary is a slight modification of Case 2 in the proof of Theorem $\mathrm{C}^{\prime}$.

Remark. It is an easy consequence of Fubini's theorem that Theorem $\mathrm{C}$ and its corollaries still hold in $\mathbf{R}^{n}$ as long as we have that the set of directions involved in the respective maximal operators lie in a fixed two-dimensional subspace of $\mathbf{R}^{n}$.

\section{REFERENCES}

[B] J. Barrionuevo, Ph.D. thesis, University of Rochester, 1990.

[CDR] M. Christ, J. Duoandikoetxea, and J. L. Rubio de Francia, Maximal operators related to the Radon transform and the Calderón-Zygmund method of rotations, Duke Math. J. 53 (1986).

[Cor 1] A. Cordoba, The Kakeya maximal function and the spherical summation multipliers, Amer. J. Math. 99 (1977).

[Cor 2] __ Geometric Fourier analysis, Ann. Inst. Fourier (Grenoble) 32 (1982).

[Cor 3] _ Maximal functions, covering lemmas and Fourier multipliers, Proc. Sympos. Pure Math., vol. 35, Amer. Math. Soc., Providence, R.I., 1979, pp. 29-50.

[Cor 4] _ , The multiplier problem for the polygon, Ann. of Math. 105 (1977).

[CF] A. Cordoba and R. Fefferman, On the equivalence between the boundedness of certain classes of maximal and multiplier operators in Fourier analysis, Proc. Nat. Acad. Sci. U.S.A. 74 (1977).

[F] C. A. Fefferman, A note on spherical summation multipliers, Israel J. Math 15 (1973).

[Ha] Young-Hwa $\mathrm{Ha}, L^{2}$-boundedness of spherical maximal operators with multidimensional parameter set, Proc. Amer. Math. Soc. 105 (1989), 401-412.

[Hor] L. Hörmander, The analysis of linear partial differential operators, Vol. I, Springer-Verlag, Berlin and New York, 1983.

[KS1] A. N. Kolmogorov and G. Seliverstov, Sur la convergence des séries de Fourier, C.R. Acad. Sci. Paris 178 (1925).

[KS2] _ Sur la convergence des séries de Fourier, Rend. Accad. Naz. Lincei 3 (1926). 
[NSW] A. Nagel, E. M. Stein, and S. Wainger, Differentiation in lacunary directions, Proc. Nat. Acad. Sci. U.S.A. 75 (1978).

[S1] E. M. Stein, Lecture Notes, Princeton Univ., 1978-1979 academic year.

[S2] _ Singular integrals and differentiability properties of functions, Princeton Univ. Press, Princeton, N.J., 1970.

[Str 1] J. Strömberg, Maximal functions associated to uniformly distributed families of directions, Ann. of Math. 108 (1978).

[Str 2] _ Weak estimates on a maximal function with rectangles in certain directions, Ark. Mat. 15 (1977).

[Z] A. Zygmund, Trigonometric series, 2nd ed., vols. 1, 2, Cambridge Univ. Press, London, New York, 1959.

Department of Mathematics, University of Rochester, Rochester, New York 14627

Current address: Department of Mathematics, University of South Alabama, Mobile, Alabama 36688

E-mail address: fjab@usouthal.bitnet 